\title{
Proposta de Monitoramento e Gerenciamento Inteligente de Temperatura em Datacenters
}

\author{
Djalma Teixeira $^{1}$, Adriano Vogel ${ }^{1,2}$, Dalvan Griebler ${ }^{1,2}$ \\ ${ }^{1}$ Laboratório de Pesquisas Avançadas para Computação em Nuvem (LARCC) \\ Faculdade Três de Maio (SETREM) - Três de Maio - RS - Brasil \\ ${ }^{2}$ Escola Politécnica, Pontifícia Universidade Católica do Rio Grande do Sul (PUCRS) \\ Porto Alegre - RS - Brasil \\ djalma.teixeira@setrem.com.br
}

\begin{abstract}
The constant growth and development of computing infrastructures has been driving an increasing demand for intelligent monitoring and management. In smart datacenters the hardware is controlled with autonomic actions that are executed under certain conditions, without the need of human intervention. The objective of this work is to propose a conceptual model of monitoring and intelligent management for temperature in smart datacenters, which can be applied in both basic and complex structures. The solution can potentially be adapted to the heterogeneity of current datacenters.
\end{abstract}

Resumo. $O$ aumento constante do crescimento e desenvolvimento das infraestruturas computacionais, vem impulsionando uma demanda cada vez maior por monitoramento e gerenciamento inteligente de datacenters. Em um ambiente gerenciado autonomicamente os equipamentos são controlados por meio de ações autonômicas, que são executadas sob determinadas condições sem a necessidade de intervenção humana. O objetivo deste trabalho é propor um modelo conceitual de monitoramento e gerenciamento inteligente para temperatura, que pode ser aplicado tanto em estruturas básicas quanto complexas $e$ adaptado a heterogeneidade dos datacenters atuais.

\section{Introdução}

O crescimento da demanda por serviços de computação em nuvem vem exigindo um aumento considerável das infraestruturas de datacenters [Vogel et al. 2016]. Segundo [Yogendra 2012], os datacenters são ambientes construídos para a concentração de servidores e equipamentos de rede, além de provisionamento de todos os serviços de TI e alto tráfego de dados. Portanto é necessário que estas infraestruturas mantenham alta disponibilidade.

Portanto estes equipamentos devem operar com climatização apropriada uma vez que possuem componentes sensíveis a temperaturas elevadas, tornando a climatização do ambiente fundamental para manter a disponibilidade dos equipamentos e serviços do datacenter. A norma [ASHRAE. 2013] define que a climatização ambiente deve se manter entre 18 e $25 \mathrm{C}$ para diminuir os riscos de superaquecimento de componentes. Deste modo para manter a temperatura ideal se faz necessário monitoramento e gerenciamento constante do ambiente e do hardware. Quando esse processo é feito de forma manual depende completamente de um operador para evitar um evento crítico. 
O principal evento crítico referente a temperatura é o superaquecimento de componentes, que pode ocasionar indisponibilidade devido a desligamentos forçados, e avariação de hardware quando exposto a longos períodos de temperatura elevada. O modelo conceitual proposto neste estudo visa servir como uma alternativa para evitar prejuízos relacionados a superaquecimento através de ações autonômicas.

Este estudo tem como objetivo propor um modelo conceitual para monitoramento e gerenciamento inteligente de temperatura, baseando-se nos requisitos abordados nas normas [TIA-942 2012] e [ASHRAE. 2013] e realizar a implementação prática do modelo conceitual utilizando a ferramenta de gerenciamento de redes Zabbix, afim de validar a eficácia da proposta.

O estudo contribui com a organização e tabulação dos requisitos descritos nas normas TIA 942 e ASHRAE referentes temperatura e climatização de datacenters. Com uma proposta de implementação e validação de modelo conceitual para monitoramento e gerenciamento inteligente de temperatura baseado em computação autonômica, e a validação prática parcial do modelo proposto.

A Seção 2 apresenta o referencial teórico deste estudo. Na Seção 3 são discutidas as normas e os requisitos para redundância e temperatura do ambiente datacenter. A Seção 4 apresenta a proposta de modelo conceitual para gerenciamento inteligente da temperatura ambiente bem como a sua validação. A Seção 5 apresenta os trabalhos relacionados deste artigo e a conclusão do artigo é mostrada na Seção6. 6.

\section{Referencial Teórico}

Esta seção explica os principais conceitos abordados neste estudo, são eles: o gerenciamento de temperatura para datacenters e a computação autonômica.

\subsection{Gerenciamento de Temperatura de datacenters}

O gerenciamento da temperatura ambiente em um datacenter tem como objetivo tratar e evitar a ocorrência de eventos críticos, como o superaquecimento por exemplo. Este processo é feito por meio do monitoramento e controle dos equipamentos de climatização e dissipação de calor dispostos na infraestrutura. Tendo em vista que os servidores possuem diversos componentes sensíveis a temperaturas elevadas o gerenciamento da temperatura em um datacenter é fundamental.

O aumento das infraestruturas computacionais exigem sistemas de climatização mais potentes para garantir a disponibilidade e a integridade dos recursos. Segundo [Mukherjee et al. 2010] os equipamentos de TI de alto desempenho concentrados nos datacenters podem em apenas alguns segundos ultrapassarem a temperatura limite definida por seus fabricantes, podendo superaquecer e comprometer os componentes e a disponibilidade dos servidores e aplicações.

\subsection{Computação Autonômica}

A computação autonômica é uma solução eficaz para o gerenciamento de grandes infraestruturas computacionais, pois possibilita o controle dos recursos sem a necessidade de intervenção humana. Segundo [Norouzi and Bauer 2015] Autonomic Computing (AC) é a capacidade de um computador de gerenciar a si mesmo automaticamente por meio 
de tecnologias adaptáveis que aprimoram os recursos de computação e reduzem o tempo exigido pelos profissionais de TI para resolver as dificuldades do sistema ou outras manutenções.

A computação autonômica visa reduzir a necessidade de intervenção humana no gerenciamento de ambientes computacionais. No âmbito de datacenters é utilizada no auto-gerenciamento, auto-reparo e na otimização dos recursos. Além disso pode ser utilizada para reduzir a complexidade no controle e na operação de infraestruturas complexas.

\section{Normas de Monitoramento de Temperatura}

Atualmente a utilização de uma infraestrutura de datacenter depende de um sistema de climatização de ambiente para manter os equipamentos operando em uma temperatura adequada, cuja a classificação e padronização é disponibilizada em algumas normas. Neste artigo foram estudadas as normas [TIA-942 2012] que classifica o nível de redundância para equipamentos de refrigeração e a [ASHRAE. 2013] que define requisitos para temperatura dentro de ambientes datacenter. No entanto estas normas não disponibilizam as informações de forma objetiva e organizada.

Portanto, as informações presentes nas normas [TIA-942 2012] e [ASHRAE. 2013] foram tabuladas e organizadas em forma de tabela para oferecer um visão mais clara dos requisitos necessários. Na tabela 1 são apresentados os requisitos para temperatura bem como a classificação considerando redundância para equipamentos de climatização de um datacenter. Os requisitos são organizados em quatro Tiers, sendo Tier 1 infraestrutura básicas, Tier 2 baixa redundância, Tier 3 alta redundância e Tier 4 a prova de falhas.

Tabela 1: Redundância de equipamentos de climatização

\begin{tabular}{|c|c|c|c|c|}
\hline Climatização & Tier 1 & Tier 2 & Tier 3 & Tier 4 \\
\hline \multicolumn{5}{|l|}{ Geral } \\
\hline $\begin{array}{l}\text { Redundância para equi- } \\
\text { pamentos mecânicos }\end{array}$ & Não requer & $\begin{array}{l}\text { Perda de refri- } \\
\text { geração }\end{array}$ & $\begin{array}{l}\text { Não causa perda } \\
\text { de resfriamento, } \\
\text { mas pode elevar } \\
\text { a temperatura }\end{array}$ & $\begin{array}{l}\text { Não causará } \\
\text { perda de resfria- } \\
\text { mento }\end{array}$ \\
\hline $\begin{array}{l}\text { Sistema de encanamento } \\
\text { de água }\end{array}$ & $\begin{array}{l}\text { Permitido mas } \\
\text { não recomen- } \\
\text { dado }\end{array}$ & $\begin{array}{l}\text { Permitido mas } \\
\text { não recomen- } \\
\text { dado }\end{array}$ & Não permitido & Não permitido \\
\hline Pressurização positiva & Não requer & Sim & Sim & Sim \\
\hline $\begin{array}{l}\text { Exige um gerador re- } \\
\text { serva }\end{array}$ & Não requer & Sim & Sim & Sim \\
\hline \multicolumn{5}{|l|}{$\begin{array}{l}\text { Sistema de refrigera- } \\
\text { ção }\end{array}$} \\
\hline $\begin{array}{l}\text { Unidades redundantes de } \\
\text { ar condicionado }\end{array}$ & Não requer & $\begin{array}{l}\text { Uma unidade de } \\
\text { AC redundante }\end{array}$ & $\begin{array}{l}\text { Qtd. de Unida- } \\
\text { des AC suficien- } \\
\text { tes para manter } \\
\text { a área crítica }\end{array}$ & $\begin{array}{l}\text { Qtd. de Unida- } \\
\text { des AC suficien- } \\
\text { tes para manter } \\
\text { a área crítica }\end{array}$ \\
\hline $\begin{array}{l}\text { Energia para sistema de } \\
\text { refrigeração }\end{array}$ & Caminho único & Caminho único & $\begin{array}{l}\text { Múltiplos cami- } \\
\text { nhos }\end{array}$ & $\begin{array}{l}\text { Múltiplos cami- } \\
\text { nhos }\end{array}$ \\
\hline Controle de umidade & Não requer & Sim & Sim & Sim \\
\hline \multicolumn{5}{|l|}{ Dissipação de calor } \\
\hline $\begin{array}{l}\text { Sistema de refrigeração a } \\
\text { água }\end{array}$ & Caminho único & Caminho único & $\begin{array}{ll}\text { Sistema } & \text { de } \\
\text { condensação } & \text { de } \\
\text { água } & \end{array}$ & $\begin{array}{ll}\text { Sistema } & \text { de } \\
\text { condensação } & \text { de } \\
\text { água } & \end{array}$ \\
\hline Liberação de calor & Caminho único & Caminho único & $\begin{array}{l}\text { Sistema de con- } \\
\text { densação a água }\end{array}$ & $\begin{array}{l}\text { Sistema de con- } \\
\text { densação a água }\end{array}$ \\
\hline
\end{tabular}




\section{Gerenciamento Autonômico de Temperatura}

Segundo [Sahana et al. 2018] o crescimento da demanda por serviços de computação em nuvem aumentou as pesquisas por soluções de gerenciamento inteligente de temperatura e energia para ambientes datacenter. Uma vez que as infraestruturas de TI venham a utilizar mais recursos computacionais a geração de calor tende a aumentar, de modo que uma falha de climatização ou dissipação de calor possa gerar consequências mais graves como desligamentos forçados e avariação de componentes.

O desenvolvimento deste modelo é baseado nas normas descritas na Seção 3 e na pesquisa realizada por trabalhos relacionados. Já o objetivo da proposta é oferecer um método para melhorar a eficácia do gerenciamento da temperatura de um datacenter através de abordagens autonômicas, sendo escalável as quatro Tiers de datacenters.

\subsection{Proposta de Modelo Conceitual}

O modelo conceitual apresentado nessa seção propõe um método de gerenciamento autonômico de temperatura em datacenter executando ações reativas quando detectada a ocorrência de eventos críticos, como por exemplo, o superaquecimento de componentes. Os eventos críticos relacionados a temperatura do ambiente e dos equipamentos de TI foram classificados da seguinte forma: (EX) se o evento tem origem externa, (IN) se o evento tem origem interna, o status que define se o evento se trata de uma (IN) informação, (AL) alerta ou (CR) estado crítico, e categoria que informa a qual categoria (T1, T2, T3 e T4) da TIA 942 o evento está direcionado .

O monitoramento desses eventos foi classificado da seguinte forma: Básico (B) monitora as funções básicas do item além de gerar gráficos e relatórios simples. Intermediário (I) monitora a maior parte dos componentes do item selecionado gera gráficos e relatórios elaborados. Avançado (A) oferece informações completas sobre os itens monitorados, ou seja, monitora todos os aspectos que podem ser trabalhados e gerenciados e mostra de forma otimizada as informações gerando gráficos, relatórios e logs.

O gerenciamento foi classificado da seguinte forma: (M) manual ações que dependem da operação de uma pessoa física, (SA) semi-autônomo pode alertar ou tentar conter a ocorrência de um evento e (AT) autônomo ações que normalizam o estado da infraestrutura. A Figura 1 apresenta o modelo conceitual de temperatura, que é dividido em temperatura de ambiente e temperatura de equipamentos. O modelo apresenta possíveis eventos elencados que podem ser monitorados e gerenciados para fins de controle autonômico.

\subsection{Implementação e Resultados}

Foi desenvolvido um fluxograma com o objetivo de representar em alto nível como as ações reagem caso ocorra o evento crítico superaquecimento dos componentes de TI em um datacenter inteligente. A Figura 2 ilustra o fluxo de verificações e decisões que devem ser tomadas quando identificado estado de risco a infraestrutura. Foram criados 4 gatilhos para disparar ações reativas no intuito de contenção de um estado crítico, ou seja, são executadas para evitar os danos decorrentes da exposição a alta temperatura, portanto se detectado um evento grave, a integridade dos equipamentos será prioridade em relação a aplicações. Os gatilhos são disparados sob a seguinte condição: 
Figura 1. Modelo Conceitual Temperatura.

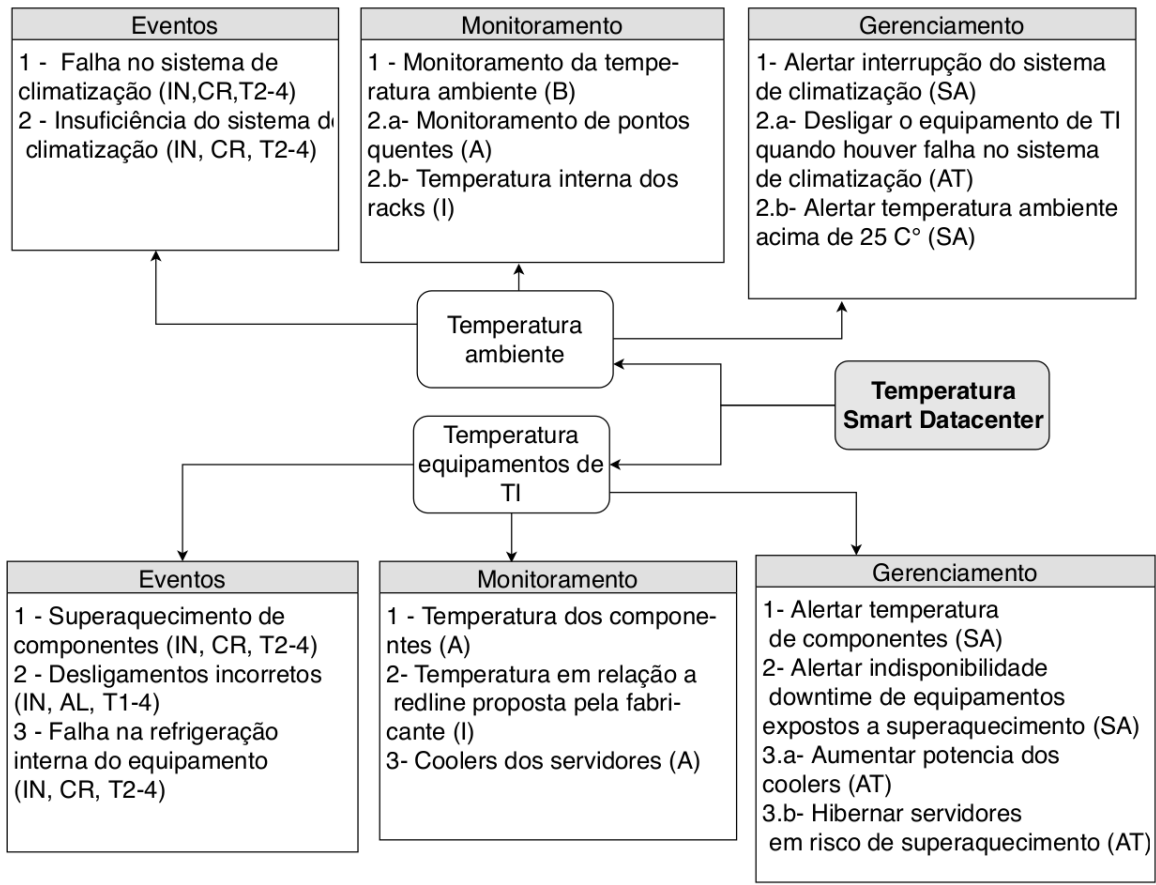

- O gatilho 1 (G1): a temperatura da CPU do host monitorado atinge o valor considerado alto pelo seu fabricante caracterizando um status de alerta.

- O gatilho (G2): executado quando o status de risco da temperatura da CPU do host monitorado está acima do valor considerado alto pelo seu fabricante.

- O gatilho 3 (G3): quando a temperatura da CPU do host monitorado atinge a redline definida pelo seu fabricante o que representa grande ameaça a integridade do componente.

- O gatilho 4 (G4): quando a temperatura reduz abaixo do status de alerta.

Também foram elaboradas ações que são executadas com o intuito de contenção, desse modo sempre que um gatilho é disparado as ações são executadas enviando um comando remoto para o host. As ações desenvolvidas para contenção do evento crítico superaquecimento foram:

- Ação 1 (A1): reduzir a frequência do processador para média performance.

- Ação 2 (A2): reduzir a frequência do processador para baixa performance.

- Ação 3 (A3): Hibernar o sistema operacional visto que os prejuízos de avariação tem alto potencial de se tornarem maiores que prejuízos decorrentes da indisponibilidade temporária.

- Ação 4 (A4): alertar redução da temperatura e normalizar o processamento através da execução de um comando remoto. Caso essa ação eleve a temperatura para o nível de alerta (G1) é disparado novamente.

- Ação 5 (A5): requer sistema de climatização gerenciável para controlar a temperatura do ambiente remotamente.

O fluxograma ilustrado na 2 tem como objetivo representar a parte do modelo referente a temperatura dos equipamentos de TI. Os gatilhos e ações foram implementados através do software [ZABBIX 2019] (versão 4.2), que é uma das ferramentas open 
Figura 2. Representação do Gerenciamento de Temperatura.

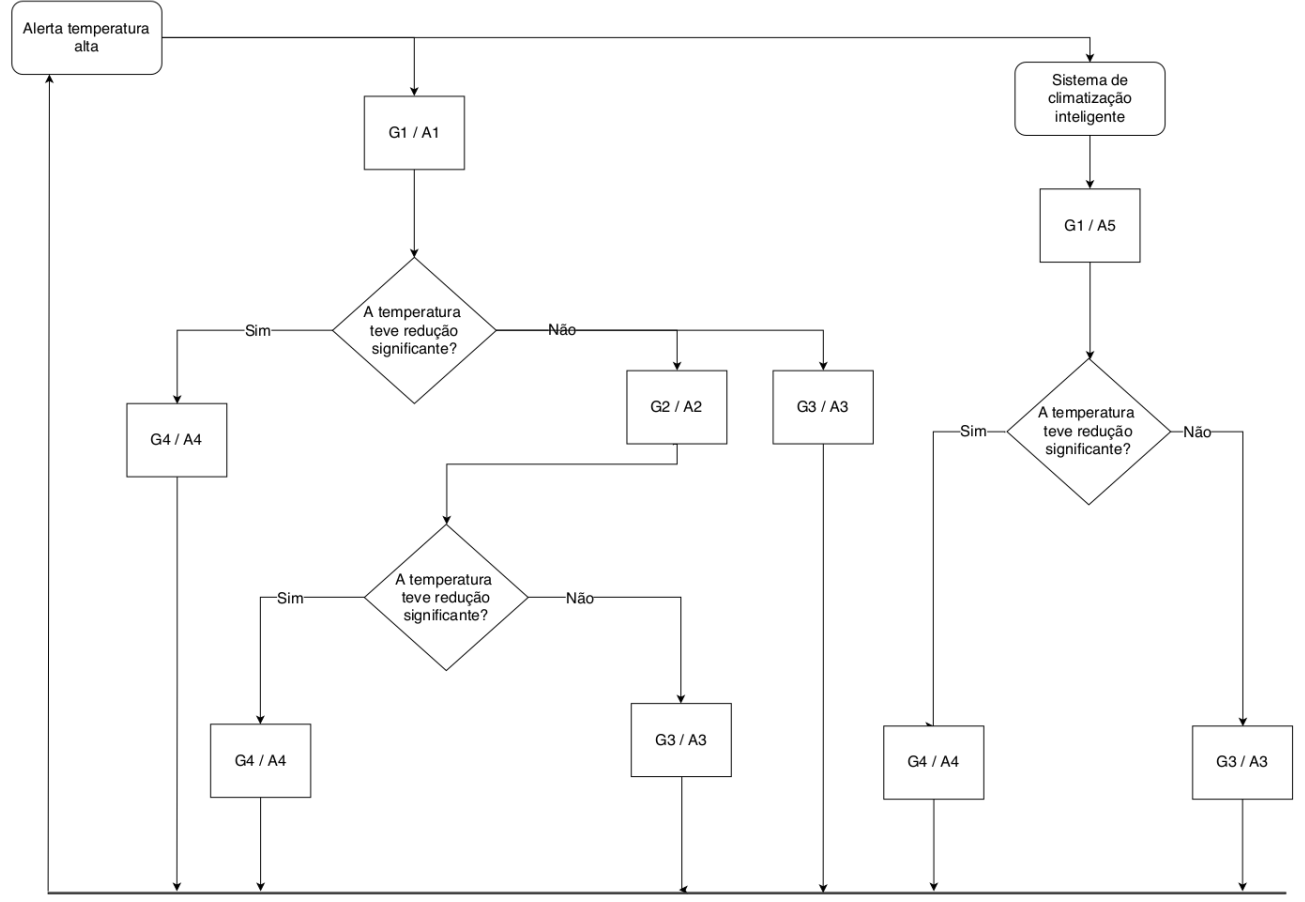

source mais robustas utilizadas para monitoramento e gerenciamento de infraestruturas computacionais, sendo instalado em um ambiente de testes no LARCC (Laboratório de Pesquisas Avançadas para Computação em Nuvem). Para o desenvolvimento das ações autonômicas foi utilizado o recurso de execução de comandos remotos da ferramenta.

O cenário utilizado neste estudo possui 4 servidores HP ProLiant DL385 G6 localizados em um dos racks do laboratório, e alocados em um ambiente climatizado. Para aplicar o modelo proposto o primeiro passo foi definir os itens que serão monitorados e analisados para tomada de decisão. Nesse experimento optou-se por monitorar a temperatura da CPU dos servidores por meio dos sensores internos do hardware.

Figura 3. Execução de ações reativas.

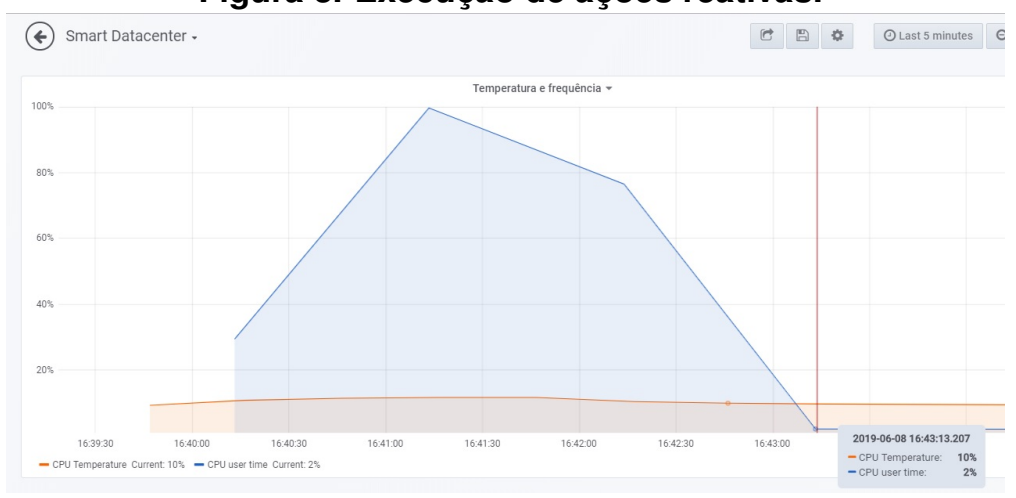

Os gatilhos foram configurados no Zabbix e servem como condição para disparo de uma ação automática. O valor para que (G1) seja verdadeiro foi alterado para $10 \mathrm{C}$ para não colocar em risco a integridade dos componentes. Quando a temperatura da CPU 
dos servidores alcança mais de $10 \mathrm{C}$ uma trigger é disparada, e a ação (A1) é executada através do comando remoto cpufreqd que reduz a frequência do processador buscando reduzir a temperatura dos componentes.

A Figura 3 ilustra o monitoramento da temperatura e carga da CPU de um dos hosts utilizados, onde o gatilho (G1) é disparado e (A1) é executada, fazendo com que a frequência do processador seja reduzida e a temperatura do componente seja diminua a um nível seguro. Vale ressaltar que caso a temperatura dos equipamentos continue aumentando até um nível crítico a ação (A3) é ativada executando o comando remoto para hibernar os servidores e controlar o evento.

\section{Trabalhos Relacionados}

O estudo de [Qu et al. 2013] tem como objetivo implantar sensores para coletar informações de temperatura, de modo a ajustar eficientemente a fonte de refrigeração do datacenter, buscando o posicionamento ideal dos sensores. O objetivo foi otimizar a carga e o consumo do sistema de refrigeração analisando os dados coletados, além de detectar e evitar pontos de aquecimento. Enquanto este artigo busca propor um modelo conceitual de monitoramento e gerenciamento autonômico para temperatura em infraestruturas de datacenter estabelecendo ações reativas para controle autonômico.

A pesquisa de [Fahrianto et al. 2017] tem como objetivo desenvolver um sistema de monitoramento inteligente baseado em IoT (Internet of Things). O estudo observou que no decorrer de um ano ocorreram incidentes no datacenter devido à instabilidade de tensão e corrente enviada a fonte de alimentação, danificando o equipamento. Enquanto neste artigo é proposto um modelo conceitual de monitoramento e gerenciamento de temperatura para Smart datacenters com objetivo de estabelecer um método de monitoramento inteligente para infraestruturas mais simples.

O estudo de [Viswanathan et al. 2011] tem como objetivo propor uma abordagem de gerenciamento autonômico para e otimização do sistema de temperatura de um datacenter através de uma rede de sensores auto-gerenciáveis, que coleta amostras do ambiente em tempo real para monitorar oscilações e anomalias dentro do ambiente datacenter, composta pelos seguintes itens: câmeras térmicas, Sensores escalares de temperatura e umidade e Sensor multicamada para medir o fluxo de ar. Enquanto este trabalho busca propor um modelo conceitual para monitoramento e gerenciamento de datacenter inteligentes como base na literatura e nas normas que estabelecem requisitos para está área.

\section{Conclusão e Trabalhos Futuros}

Com a realização deste estudo buscou-se propor um modelo que possa contribuir com a implementação do gerenciamento inteligente da temperatura nas atuais infraestruturas de datacenter. Através da análise dos resultados percebeu-se que o modelo proposto tem maior eficácia quando as ações autonômicas são executadas de forma reativa com objetivo de conter eventos críticos, como por exemplo, reduzir recursos quando necessário a fim de priorizar a integridade dos equipamentos de TI. Com os resultados concluiu-se que a execução das ações autonômicas podem reduzir a temperatura dos equipamentos de TI através da redução da frequência do processador, a medida que os níveis de calor dos componentes aumentar. 
O modelo poderia ser aplicado em infraestruturas de maior porte, desde que os equipamentos possam ser monitorados e que haja dispositivos gerenciáveis que suportem a execução de comandos remotos, pois a solução tende a ser mais precisa com um maior número de dados coletados. Exitem outras áreas em um datacenter que demandam de um gerenciamento inteligente, portanto para a realização de trabalhos futuros fica o desenvolvimento de um modelo conceitual para monitoramento e gerenciamento inteligente de energia, recursos computacionais, telecomunicações e segurança, bem como a utilização de uma rede de sensores de temperatura ambiente para aprimorar o modelo proposto neste artigo.

\section{Agradecimentos}

O presente trabalho foi realizado com apoio da Coordenação de Aperfeiçoamento de Pessoal Nível Superior - Brasil (CAPES) - Código de Financiamento 001

\section{Referências}

[ASHRAE. 2013] ASHRAE. (2013). 2013 ASHRAE Handbook: Fundamentals. ASHRAE.

[Fahrianto et al. 2017] Fahrianto, F., Anggraini, N., Suseno, H. B., Shabrina, A., and Reza, A. (2017). Smart data centre monitoring system based on Internet of Things (IoT). In Int. Conf. on Cyber and IT Service Management (CITSM), pages 1-9, Indonesia. IEEE.

[Mukherjee et al. 2010] Mukherjee, T., Banerjee, A., Varsamopoulos, G., and Gupta, S. K. (2010). Model-driven coordinated management of data centers. Computer Networks, 54(16):2869-2886.

[Norouzi and Bauer 2015] Norouzi, F. and Bauer, M. (2015). Autonomic management for energy efficient data centers. CLOUD COMPUTING 2015, page 153.

[Qu et al. 2013] Qu, J., Li, L., Liu, L., Tian, Y., and Chen, J. (2013). Smart temperature monitoring for data center energy efficiency. In IEEE Int. Conf. on Service Operations and Logistics, and Informatics, pages 360-365, Dongguan, China. IEEE.

[Sahana et al. 2018] Sahana, S., Bose, R., and Sarddar, D. (2018). Server utilization-based smart temperature monitoring system for cloud data center. In Industry Interactive Innovations in Science, Engineering and Technology, pages 309-319. Springer.

[TIA-942 2012] TIA-942 (2012). Tia-942 data center standards overview.

[Viswanathan et al. 2011] Viswanathan, H., Lee, E. K., and Pompili, D. (2011). Selforganizing sensing infrastructure for autonomic management of green datacenters. Ieee Network, 25(4).

[Vogel et al. 2016] Vogel, A., Griebler, D., Maron, C. A. F., Schepke, C., and Fernandes, L. G. (2016). Private IaaS Clouds: A Comparative Analysis of OpenNebula, CloudStack and OpenStack. In Euromicro Int. Conf. on Parallel, Distributed and NetworkBased Processing (PDP), pages 672-679, Heraklion, Greece.

[Yogendra 2012] Yogendra, Joshi Kumar, P. (2012). Energy efficient thermal management of data centers. Springer Science \& Business Media.

[ZABBIX 2019] ZABBIX (2019). Zabbix documentation. Disponível em: http://www.zabbix.com/. Acesso em: 15 Fevereiro de 2019. 